\title{
Nonaxisymmetric cool spot distributions and dynamo action in close binaries
}

\author{
D. $\operatorname{Moss}^{1}$, N. Piskunov ${ }^{2}$, and D. Sokoloff ${ }^{3}$ \\ 1 Department of Mathematics, Manchester University, Manchester M13 9PL, UK \\ 2 Institute of Astronomy and Space Physics, Uppsala University, 75120, Uppsala, Sweden \\ e-mail: piskunov@astro.uu.se \\ 3 Department of Physics, Moscow State University, 119899, Moscow, Russia \\ e-mail: sokoloff@dds.srcc.msu.su
}

Received 8 July 2002 / Accepted 19 September 2002

\begin{abstract}
We investigate the nonaxisymmetric magnetic field distribution in the components of close binary systems, generated by a stellar dynamo with a nonaxisymmetric distribution of the $\alpha$-coefficient, proportional to the mean helicity of the convective motions in the stellar convective envelope. The nonaxisymmetry of $\alpha$ is assumed to be connected with interaction with the binary companion, i.e. the reflection effect and tidal interaction. The degree of asymmetry is estimated from the known parameters of ER Vulpeculae, an active RS CVn-type star. We demonstrate that the synchronization of rotation and orbital motion of ER Vulpeculae due to tidal interaction is sufficient to allow nonaxisymmetric magnetic structures to survive in the presence of smoothing by differential rotation. The dynamo driven nonaxisymmetric magnetic structures are usually steady in the reference frame connected with the $\alpha$-spot. However, we found a resonant excitation of an oscillatory, strongly nonaxisymmetric, configuration, driven by the joint action of the $\alpha$-effect and differential rotation. The magnetic structures obtained are predominantly manifested on the stellar surface in the form of spots of radial magnetic field. Their form and size are similar to the form and size of cool spots observed on the surfaces of both components of ER Vulpeculae, and their displacements from the $\alpha$-spots are comparable to that of the observed cool spots from the hot spots connected with the reflection effect. Possible relevant observations are discussed.
\end{abstract}

Key words. stars: activity - MHD - stars: binaries: general - stars: magnetic fields

\section{Introduction}

Many late-type stars in close binary systems, e.g. the RS CVn group, have manifestations of magnetic activity which seems to be persistently nonaxisymmetric (e.g. Piskunov et al. 1990; Henry et al. 1995; Olah et al. 1997). The non-axisymmetric nature of stellar activity in close binaries is much more pronounced than that discussed in connection with the possible existence of active longitudes for the solar activity (see e.g. Jetsu et al. 1997). The magnetic activity of late-type stars is thought to be connected with dynamo action. The aim of this paper is to discuss the nonaxisymmetric nature of the large scale magnetic fields of close binaries as a result of nonaxisymmetric dynamo action; we base our analysis on the example of an active RS CVn type binary ER Vulpeculae. The system consists of two late-type main sequence stars $\left(T_{\text {eff }}=5800\right.$ and $5500 \mathrm{~K}, R_{*}=1.32$ and $1.27 R_{\odot}$ ), separated by $4.2 R_{\odot}$, the orbital period is $0.698 \mathrm{~d}$ and the system appears to be rotationally synchronized (Gunn \& Doyle 1997). Doppler images of the system have been obtained by Piskunov (1996) and Piskunov et al. (2001) for two observational seasons (1994 and

Send offprint requests to: D. Moss, e-mail: moss@ma.man.ac.uk
1996); see Sokoloff \& Piskunov (2002) for a discussion of the observational situation from the viewpoint of dynamo theory.

The distribution of spots on ER Vulpeculae appears to be remarkably nonaxisymmetric. Dark spots, which are thought to be connected with magnetic activity, seem to be anchored to the bright spot associated with the reflection effect. The dark spots are located in polar and subpolar latitudes, and their form appears to be quite variable with time, so it would be natural to associate them with an oscillating dynamo solution, with magnetic field concentrated in the subpolar regions. This form of spot structure seems to be intrinsically connected with the tidal interaction between two members of the close binary system. When considering similar systems, there appears to be a gradual reduction of the apparent degree of nonaxisymmetry with increasing stellar separation. Long-term monitoring of active regions on ER Vul with Doppler Imaging techniques (Piskunov et al. 2001), HR 1099 (Vogt et al. 1999) and II Peg (Berdyugina et al. 1999) shows a progressive increase in drift velocity of the active regions with respect to the line connecting the binary components. ER Vul which has the smallest separation between its components shows no such drift. On the other hand, the separation of the components of ER Vulpeculae is large enough 
to exclude direct magnetic flux exchange between the primary and secondary components.

This conclusion, being based on only three examples, obviously needs additional confirmation. Hall (1991) studied the connection between the Roche-lobe filling factor and a differential rotation coefficient, and found that differential rotation decreases as the stellar separation decreases. Such a variation in differential rotation is certainly consistent with our idea. We stress that additional observations to clarify the relations between stellar separation, differential rotation and nonaxisymmetric structures would be more than welcome.

Because a binary system is intrinsically nonaxisymmetric the idea of nonaxisymmetric dynamo action in such a system prima facie appears quite natural. Of course, heating by the reflection effect as well as by tidal forces can result in a nonaxisymmetric distribution of the dynamo control parameters (for example, the coefficient $\alpha$, which is proportional to the helicity). We present the relevant estimates in Sect. 2. We demonstrate in Sect. 4 that the associated magnetic field distribution has a substantial nonaxisymmetry, whose geometrical shape is quite similar to that of the observed dark spots. This result persists even in the presence of a degree of differential rotation; we allow the $\alpha$-spot to corotate with the stellar envelope at various depths below the surface, so that the spot moves with respect to the stellar surface. The timescale connected with this motion is much longer than the orbital period (about 1 day) of these binary systems, because the system is synchronized by tidal interaction. However the dynamo timescale (of order 10 years) is in turn much longer than the orbital timescale. So an obvious question to be clarified is to what extent the differential rotation can coexist with a nonaxisymmetric structure generated by an $\alpha$-spot (see Sect. 5).

The following more delicate problem is connected with the temporal behaviour of a nonaxisymmetric solution. The form of the observed dark spot appears to be consistent with the idea of a nonoscillatory dynamo solution, and a standard dynamo for a basically rigid rotating body, i.e. the socalled $\alpha^{2}$-dynamo, usually does give nonoscillatory solutions. Sokoloff \& Piskunov (2002) suggested a swing exitation between a slowly rotating $\alpha$-spot and an oscillating $\alpha^{2} \omega$-dynamo mode, driven by the joint action of helicity and differential rotation, and which is subcritical in a nonresonant case. We demonstrate in Sect. 6 that for some rotation laws a resonant excitation of oscillating magnetic structure occurs, and the geometrical shape of such "spots" remains similar to that of the observed spot structure.

\section{Nonaxisymmetric dynamo drivers in close binaries}

We present in Table 1 the data concerning the spots on ER Vul that can be clearly identified from observations performed in 1994 or 1996. If a spot was observed in both observational periods, the first entry corresponds to the year 1994 and the second to the year 1996. We note that the identification of spots based on only two observational periods separated by two years contains some uncertainty. A more systematic monitoring of stellar activity, say several times per year, would obviously be helpful.
Table 1. Summary of observed spot parameters for ER Vulpeculae. Spots on the primary component are identified with upper case letters, those on the secondary with lower case. Temperatures $(T)$ are in Kelvin. Spots A, X, Z, a, c, x, y were clearly observable in 1994, spots A, B, X, Y, Z, a, b, x were clearly observable in 1996.

\begin{tabular}{lll}
\hline \hline Spot & Size $(\mathrm{deg})$ & $T$ \\
\hline Primary: & $T_{\text {mean }}=5800 \mathrm{~K}$ \\
\hline $\mathrm{A}$ & $30 \ldots 15$ & $4900 \ldots 5010$ \\
$\mathrm{~B}$ & 22 & 4980 \\
$\mathrm{X}$ & 22 & 4980 \\
$\mathrm{X}$ & $30 \ldots 15$ & $6190 \ldots 6240$ \\
$\mathrm{Y}$ & 12 & 5980 \\
$\mathrm{Z}$ & $17 \ldots 9$ & $6150 \ldots 5990$ \\
\hline Secondary: $T_{\text {mean }}=5500 \mathrm{~K}$ \\
\hline $\mathrm{a}$ & $28 \ldots 14$ & $4520 \ldots 4590$ \\
$\mathrm{c}$ & 10 & 4650 \\
$\mathrm{x}$ & $22 \ldots 20$ & $5590 \ldots 5610$ \\
$\mathrm{y}$ & 18 & 5510 \\
\hline
\end{tabular}

However it looks quite plausible that at least one pair of spots on each star, one hot (X and $\mathrm{x}$ ) and one cold (A and a) persist throughout the span of the observations.

The simplest estimate of the temperature inhomogeneity is $\delta T=\left(T_{\text {max }}-T_{\text {mean }}\right) / T_{\text {mean }}$, where the maximal temperature $T_{\max }$ corresponds to the spot $\mathrm{X}$ for the primary and to the spot $\mathrm{x}$ for the secondary, results in a rather low estimate $\delta T \approx 6 \%$. Taking temperatures $T_{\min }$ from the spots $\mathrm{A}$ and a for the primary and secondary respectively, we conclude that it is reasonable to consider values of $\delta T$ as large as $25 \%$.

An estimate for the tidal force can be based on the ratio $\delta R=R_{*} / \Delta R$, where the stellar radius $R_{*}=1.32,1.27 R_{\odot}$ for the primary and secondary components respectively, and the stellar separation is $\Delta R=4.2 R_{\odot}$. We believe that the intensity of perturbation of dynamo control parameters directly connected with tidal perturbations is governed by $\delta R$.

The spatial size of $\alpha$-spots arising from the reflection effect is controlled by $\delta R$, and their intensity is expected to decrease with increasing $\delta R$. The tidal perturbations have quadrupolar symmetry and can have much larger spatial extent than the temperature spots; however their intensities decrease as $\delta R$ grows. We conclude that the following results have a threshold nature and occur only for stars with sufficiently small values of $\delta R$.

Our model below contains only an alpha-quenching nonlinearity. Another possibility, which might be more favourable for promoting the generation of nonaxisymmetric magnetic structures, is the feedback of the Lorentz force torques onto the differential rotation (e.g. Moss \& Brooke 2000). The differential rotation plausibly can be modified by these torques into a form that is less effective in destroying nonaxisymmetric fields.

Given that the evaluation of the nonaxisymmetry of the helicity is a quite delicate problem we take, as a crude estimate, that in our alpha-quenched model the effective relative alpha perturbations caused by the helicity variations arising from each perturbation, i.e. the reflection effect and tidal interactions, may be as large as $\delta T$ and $\delta R$, i.e. $\delta \alpha=\left(\alpha_{\max }-\right.$ $\left.\alpha_{\min }\right) / \alpha_{\mathrm{av}} \approx 25 \%$. Here $\alpha_{\mathrm{av}}$ is a typical value of the helicity 
outside the spots. We assume quite arbitrarily that the contributions of the reflection effect and tidal interactions to the $\alpha$-perturbation are additive.

Then, considering the joint effects of helicity perturbations and tides, in our computed examples we consider a total perturbation $\delta \alpha=50 \%$ as a realistic but crude estimate for the magnitude of the $\alpha$ inhomogeneity, and $\delta \alpha=100 \%$ as a still acceptable estimate. We take as a crude estimate for the angular size of the $\alpha$-spots the value $\delta R$, and so we conclude that the size of $\alpha$-spots can reach $40^{\circ}$. We investigate some other values of the amplitude and size for $\alpha$-spot for orientation.

The mean hydrodynamical parameters such as $\alpha$ can be essentially modified by an external forcing on the timescale of a turnover time at least. It means that the $\alpha$-spot can be pronounced, provided that the rotation of the binary system components and their orbital motion is sufficiently synchronized by tidal interactions, i.e. again the stellar separation should be quite small. For ER Vulpeculae the "hot spot" position is observed to be stable on the timescales of years (Sokoloff \& Piskunov 2002).

\section{The dynamo model}

Our dynamo model is based on the standard equation of meanfield electrodynamics (see e.g. Krause \& Rädler 1980)

$\frac{\partial \boldsymbol{B}}{\partial t}=\nabla \times(\alpha \boldsymbol{B}+\boldsymbol{V} \times B-\eta \nabla \times \boldsymbol{B})$,

where $\boldsymbol{B}$ is the large-scale magnetic field, $\boldsymbol{V}$ is the velocity associated with the differential rotation, $\eta$ is the turbulent diffusivity (assumed to be spatially homogeneous throughout the convective shell) and $\alpha$ describes the conventional $\alpha$-effect proportional to mean helicity. (Note that in this paper we restrict ourselves to discussing the large-scale part of the magnetic field.) Because the separation of the binary systems under discussion is large enough to exclude a direct magnetic flux interchange, we consider magnetic field generation in the envelope of each component of a binary system separately, describing effects of the companion only by perturbations of the dynamo governing values. We consider a perturbation of $\alpha$ only, while it is natural to believe that the other crucial ingredients, such as $\eta$ and $\boldsymbol{V}$, can also be perturbed. Nevertheless, we here consider the turbulent diffusivity and rotation curve to be given, and include the back-reaction of the magnetic field on the fluid motion in the form of an $\alpha$-quenching, taking the quenching function to be homogeneous through the convective shell. In principle, a perturbation to the quenching function caused by the companion star could also be considered.

We use a slightly modified form of the nonaxisymmetric mean field dynamo code of Moss et al. (1991a). This code solves the mean field dynamo problem, by time stepping on a finite difference grid over spherical polar coordinates $r, \theta$, with a Fourier expansion over the longitude $\phi$. (Note that $\theta$ is measured from the "north" pole; we also introduce the latitude $\Theta$.) A naive application to nonaxisymmetric distributions of $\alpha$ was made by Moss et al. (1991b), and Moss \& Tuominen (1997) studied nonaxisymmetric $\alpha^{2}$-dynamo solutions in the context of very close or contact binary systems. The code is here applied to a spherical shell with normalized radial extent $r_{0} \leq r \leq 1$; we consider only $r_{0}=0.64$ here. Vacuum boundary conditions are applied at $r=1$, and at the base of the "convective zone" $r=r_{0}$ we used an "overshoot" condition, simulating fields falling to zero at $r \approx r_{0}-\delta$ (cf. Moss et al. 1990). We took $\delta=0.03$, but experience shows that solutions are not very sensitive to modest changes in this value. We found that for the modestly supercritical parameters investigated here, a spatial grid with 21 points uniformly distributed over $r_{0} \leq r \leq 1$, and 41 points over $0 \leq \theta \leq \pi$, with Fourier expansions to order $m=5$, adequately resolved our solutions. For an illustration of the longitudinal resolution of the code, we show in Fig. 1 the $\phi$-dependence of a function $f$ chosen later (see Sect. 4) to describe latitudinal modulations of $\alpha$ and its Fourier representation as implemented. We give a formal definition of $f$ in Sect. 4.

The spatial resolution of the code is comparable with the observational resolution available for EU Vulpeculae. Spots of spatial size of tens of degrees can be resolved; simulation of stellar dynamos on a spatial scale sufficient to resolve individual active regions as small as found on the Sun, for example, would require much more extensive computations, and a much more detailed description of the relevant physical processes.

We measure length in units of stellar radius $R$, with typically $R \approx 10^{11} \mathrm{~cm}$, and time in units of the global diffusion time $t_{\mathrm{d}}=R^{2} / \eta$, where $\eta$ is the turbulent diffusivity. If we use for estimates the solar value $\eta=\eta_{\odot} \approx 10^{12} \mathrm{~cm}^{2} \mathrm{~s}^{-1}$, then $t_{\mathrm{d}}$ is about $300 \mathrm{yr}$. This timescale looks too long to be associated with the observed timescales of stellar activity. We conclude that the turbulent diffusivity should be taken as about $\eta=30 \eta_{\odot}$ to get interesting results with $t_{\mathrm{d}} \approx 10 \mathrm{yr}$.

The intensity of the dynamo sources, $\alpha$ and differential rotation, are measured by the dimensionless numbers $C_{\alpha}=$ $f_{\alpha} \alpha_{0} R / \eta$ and $C_{\omega}=\Omega^{\prime} R^{3} / \eta$, where $\alpha_{0}$ is a scale for $\alpha$, defined below, and $\Omega^{\prime}$ is a typical value of the rotational shear, $\eta$ is the (uniform) turbulent diffusivity and $R$ is the stellar radius. Here $f_{\alpha}$ is a number of order unity that depends weakly on $\delta \alpha$ ( $f_{\alpha}=1,1.09,1.18$ for $\delta \alpha=0,0.5,1.0$ respectively). Its introduction is a technical consequence of the manner in which the nonaxisymmetric perturbation (see Sect. 4) was implemented in the code.

\section{Nonaxisymmetric structures generated by alpha-spots}

We start by considering a binary system in which tidal interactions lead to a complete synchronisation of stellar rotation, so the rotation becomes rigid, i.e. we consider a dynamo driven by mean helicity only, the $\alpha^{2}$-dynamo.

Using spherical polar coordinates $r, \theta, \phi$, we consider an $\alpha$-perturbation that is centred at the equator, $\theta=90^{\circ}$, and at $\phi=0^{\circ}$, with no radial dependence. The value $\delta \alpha=1$ corresponds to a doubling of $\alpha$ at the centre of the spot.

We take as a nonperturbed $\alpha$-profile $\alpha_{0}=\sin ^{2} \theta \cos \theta$. We adopted this form rather than the more $\operatorname{common} \cos \theta$ dependence, because with a $\phi$-dependent alpha effect it is necessary that $\alpha_{0}=0$ at the poles to avoid singularities. (This is a well known formal problem of the dynamo equations, but only 


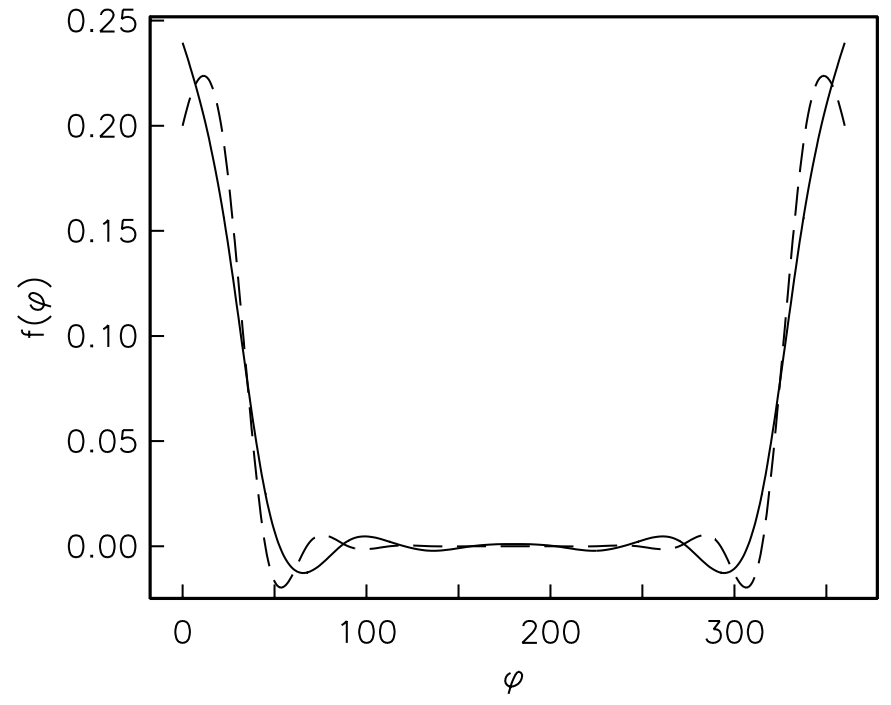

Fig. 1. Spatial modulation of the $\alpha$-coefficient. The $\phi$ dependence of the function $f$ (broken line) and its Fourier representation with 5 harmonics (solid line) are shown. Here $\Delta \phi=20^{\circ}$ - see discussion in Sect. 4.

Table 2. Summary of results for the $\alpha^{2}$ models. Here $C_{\alpha}=26$, except for entries with superscript $\dagger$, where $C_{\alpha}=30$. The values of the global nonaxisymmetry parameter $M$ (see text) are tabulated.

\begin{tabular}{llll}
\hline \hline$\delta \alpha$ & 0.2 & 0.5 & 1.0 \\
\hline$\Delta \theta$ & & & \\
\hline $22.5^{\circ}$ & & & $0.024^{\dagger}$ \\
$45^{\circ}$ & & 0.27 & 0.55 \\
$80^{\circ}$ & $0.08^{\dagger}$ & 0.40 & 0.91 \\
\hline
\end{tabular}

causes numerical difficulties when $\alpha$ depends explicitly on longitude.) Thus our $\alpha$-profile is given by $\alpha=\alpha_{0}(\theta)(1+f(\theta, \phi))$, with $f=0$ when $|\theta-\pi / 2|>\Delta \theta$ and when $|\phi|>2 \Delta \phi$. Note that $\theta=\pi / 2$ corresponds to the stellar equator. (The dimensional amplitude $\alpha_{0}$ occurring in the $\alpha$-profile is included in the definition of the dimensionless number $C_{\alpha}$.) When $|\phi|<\Delta \phi$, then $f=\delta \alpha$, with a smooth transition between 0 and $\delta \alpha$ in the interval $(\Delta \phi, 2 \Delta \phi)$. In the $\theta$-direction, there is a jump in the value of $f$ at $\theta-\pi / 2= \pm \Delta \theta$. (In test integrations this rather crude description was found to give acceptable numerical behaviour and to reproduce closely results with a smooth $\theta$-dependence.) We choose for the sake of definiteness $\Delta \phi=20^{\circ}$. Some results of our numerical experiments are summarized in Table 2. Here the global degree of nonaxisymmetry, $M$, is defined as the ratio of the energy contained in the nonaxisymmetric part of magnetic field to the total magnetic energy.

The marginal value of $C_{\alpha}$ is about 22-26, depending on the value of $\delta \alpha$ and $\Delta \theta$. Note that with the intrinsically nonaxisymmetric $\alpha$-distribution studied, it is not possible to consider axisymmetric and single mode nonaxisymmetric solutions separately - they are intrinsically intermingled. We choose slightly supercritical values, $C_{\alpha}=26$, or in cases where the critical value of $C_{\alpha}$ was larger, $C_{\alpha}=30$ (these cases are indicated by ${ }^{\dagger}$ in Table 2). Increasing the degree of supercriticality (i.e. the magnitude of $C_{\alpha}$ ) with the other parameters fixed results in
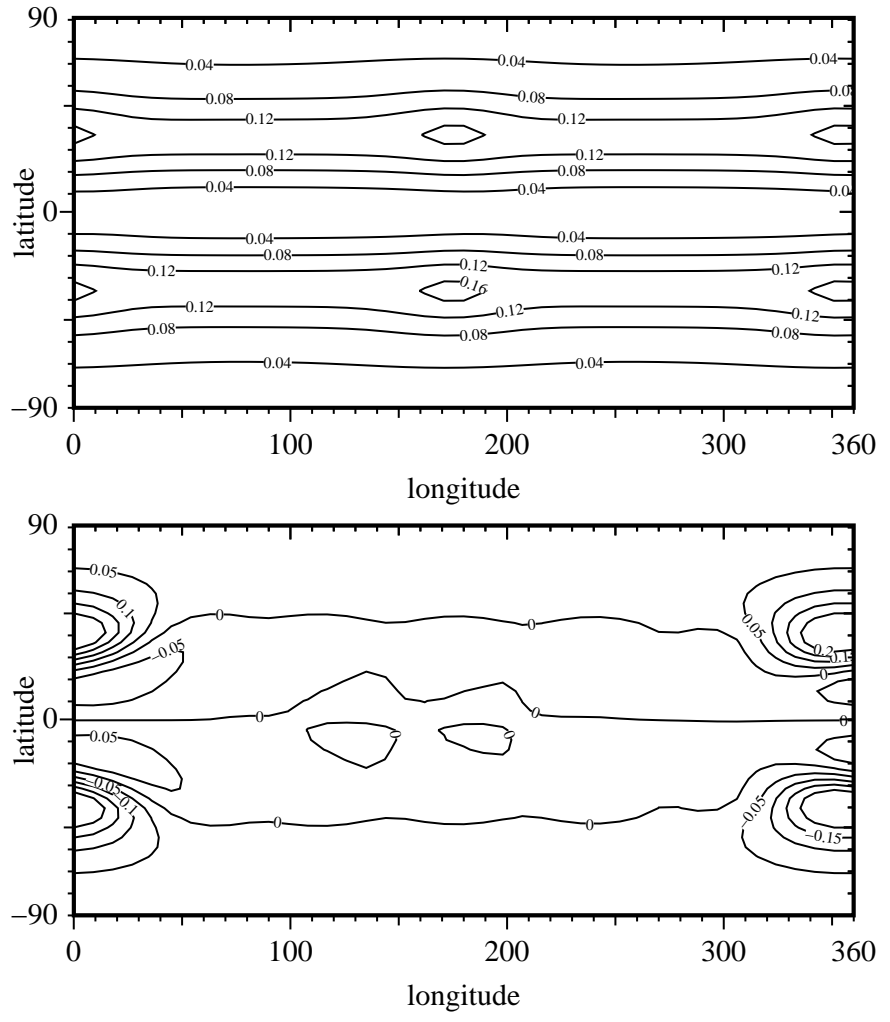

Fig. 2. Magnetic field structure at the stellar surface for a rigidly rotating close binary component (i.e. an $\alpha^{2}$ dynamo); isolines of surface large scale magnetic field distribution are shown for total magnetic field (top), and the radial component (bottom), for $\delta \alpha=1, \Delta \theta=45^{\circ}$, $C_{\alpha}=26$. The axes are labelled in degrees of latitude and longitude.

smaller values of $M$ (see Table 5). Cases where $M$ can be predicted to be very small are indicated by blank entries in Table 2 . In all cases the solution is steady with dipole-like (odd) symmetry. We conclude that an enhancement of helicity of order $50 \%$ in the spot is sufficient to obtain substantially nonaxisymmetric magnetic structures.

Typical magnetic field configurations are presented in Fig. 2. A peculiarity of these and other solutions discussed later is that the nonaxisymmetric structure at the surface is much stronger in the radial magnetic field than in the total magnetic field. Even the solutions with relatively small values of $M$ show quite noticeable spots in $B_{\mathrm{r}}$. This structure of the spot distribution is typical of all the following cases. Below we show its most important variations only. The spots are located near longitude $\phi=0^{\circ}$, close to mid-latitudes $\Theta= \pm 45^{\circ}$ (i.e. $\Theta=0^{\circ}$ corresponds to the equator). The spots are displaced towards the subpolar regions if an $\alpha$-profile that extends more to the polar regions is used (for example, if we use instead of $\alpha_{0}=\sin ^{2} \theta \cos \theta, \alpha_{0}=\cos \theta$ for $20^{\circ} \leq \theta \leq 160^{\circ}$ and $\alpha_{0}=\cos \theta \sin ^{2} \theta / \sin ^{2} 20^{\circ}$ otherwise, then the spots are located at about latitudes $\Theta= \pm 70^{\circ}$ ).

\section{The role of differential rotation}

Differential rotation could be expected to lead to some smoothing of the nonaxisymmetry of the magnetic field distribution, provided that no resonant excitation occurs. We investigate 
Table 3. Summary of results for the $\alpha^{2} \omega$ models, $\Omega=C_{\omega}\left(r-r_{0}\right)$, with $\Delta \theta=45^{\circ}, C_{\alpha}=26 . M$ and $P$ are the global nonaxisymmetry and parity parameters, $M_{\mathrm{r}}, M_{\theta}$ and $M_{\phi}$ indicate the contribution to $M$ from each magnetic field component, $E$ is the total energy of large scale magnetic field measured in units of equipartition energy. For $C_{\omega}= \pm 250$, the dynamo is not excited with these parameters. The values of $\phi$ indicate the approximate central longitudes for the dominant $B_{\mathrm{r}}$-spots, in degrees.

\begin{tabular}{llllllll}
\hline \hline$C_{\omega}$ & $M$ & $M_{\mathrm{r}}$ & $M_{\theta}$ & $M_{\phi}$ & $P$ & $E$ & $\phi$ \\
\hline 100 & 0.31 & 0.09 & 0.09 & 0.13 & -1.0 & 0.024 & 26 \\
50 & 0.31 & 0.10 & 0.09 & 0.13 & -1.0 & 0.044 & 16 \\
25 & 0.36 & 0.11 & 0.10 & 0.15 & -1.0 & 0.050 & 12 \\
0 & 0.55 & 0.16 & 0.20 & 0.18 & -1.0 & 0.045 & -5 \\
-15 & 0.51 & 0.14 & 0.21 & 0.16 & -1.0 & 0.040 & -12 \\
-25 & 0.51 & 0.13 & 0.20 & 0.18 & +1.0 & 0.042 & -17 \\
-50 & 0.52 & 0.12 & 0.19 & 0.17 & +1.0 & 0.038 & -21 \\
-100 & 0.52 & 0.11 & 0.19 & 0.21 & +1.0 & 0.021 & -31 \\
\hline
\end{tabular}

here to what extent the presence of differential rotation is compatible with the existence of nonaxisymmetric structure.

\subsection{Rotation law $\Omega(r)$}

Because our knowledge of the spatial dependence of angular velocity in close binaries is poor, we choose a simple differential rotation law $\Omega=\Omega(r)=C_{\omega}\left(r-r_{\omega}\right)$. We are thus working in a frame corotating with the radius $r_{\omega}$. As the basis for our investigation we took $r_{\omega}=r_{0}$, so that in this case the spot corotates with the bottom of the dynamo region. Taking $C_{\alpha}=26$, $\delta \alpha=1, \Delta \theta=80^{\circ}, \Delta \phi=22.5^{\circ}$ gives the results listed in Table 3.

We define $P$ as the global solution parity, see Moss et al. (1991a). $P=-1$ corresponds to a solution with $B_{\mathrm{r}}$ odd with respect to the equatorial plane (e.g. an axisymmetric dipole), whilst $P=+1$ corresponds to solutions with even symmetry of $B_{\mathrm{r}}$ with respect to the equator, i.e. a quadrupole with axis parallel to the rotation axis (in principle, more complicated configurations like a dipole with perpendicular axis are possible). We stress that here and below the parity of the solutions is broadly determined by the sign of $C_{\omega}$, with the transition between $P=+1$ and $P=-1$ occurring in the range $-20 \leq C_{\omega} \leq-15$. There does not appear to be a significant window of stable mixed mode solutions separating the two regimes. The magnetic structures obtained do not migrate in the frame of the imposed $\alpha$-spots.

Magnetic field structures corresponding to $C_{\omega}= \pm 50$ are presented in Figs. 3a, b. The spots of radial magnetic field are very similar to those presented in Fig. 2, but the location of the spots is slightly altered by the differential rotation. Remarkably, we usually find that magnetic spots of total magnetic field are near $\phi=0^{\circ}$ and $\phi=180^{\circ}$. Again, the nonaxisymmetric structures are much more pronounced in radial magnetic field. To quantify the contributions to the global nonaxisymmetry from the various magnetic field components, we introduce the quantities $M_{\mathrm{r}}, M_{\theta}$ and $M_{\phi}$ representing ratios of magnetic energies containing in corresponding nonaxisymmetric parts of
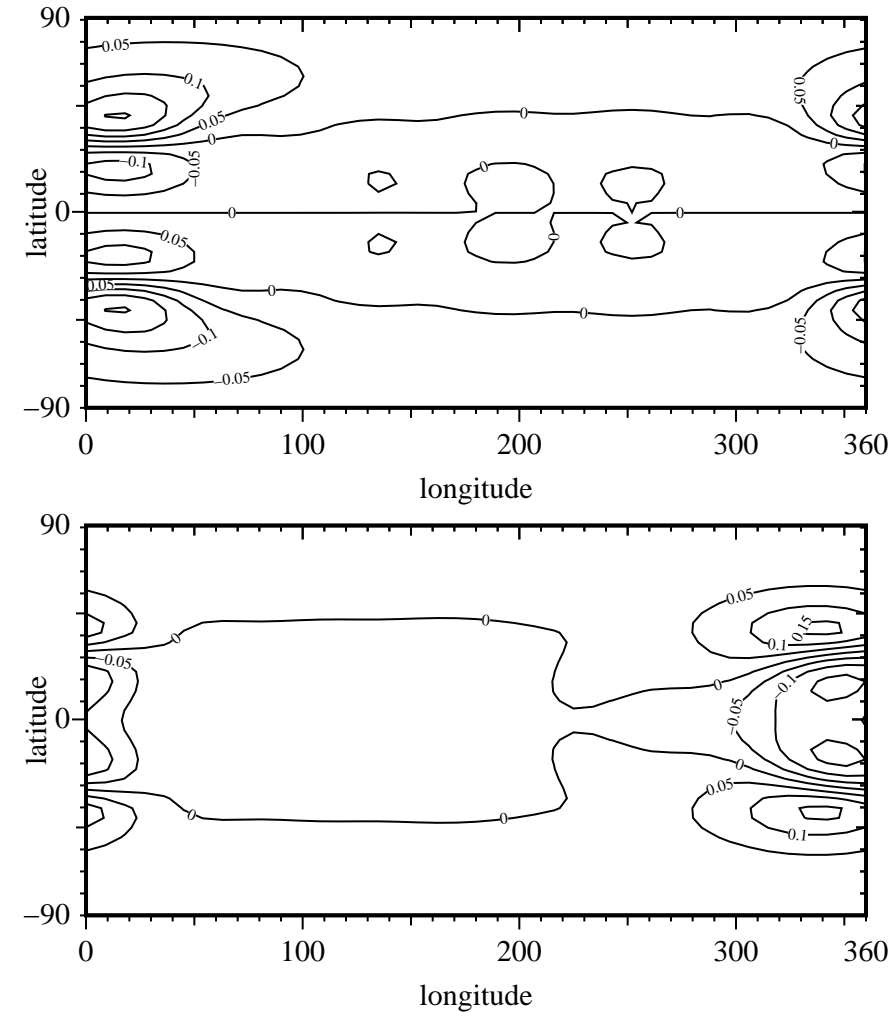

Fig. 3. Magnetic field structure at the stellar surface for a typical steady $\alpha^{2} \omega$ model, $C_{\alpha}=26, \Delta \theta=45^{\circ}, \delta \alpha=1$; isolines of radial magnetic field for a) $C_{\omega}=+50$ (top) and b) $C_{\omega}=-50$ (bottom).

Table 4. Further results for $\alpha^{2} \omega$ dynamo models, $\Omega=C_{\omega}\left(r-r_{0}\right)$, showing the dependence of the nonaxisymmetry parameters $M_{\mathrm{r}}, M_{\theta}$, $M_{\phi}$ on the model parameters $\Delta \theta, \delta \alpha$, when $C_{\alpha}=26, C_{\omega}=50 . P=-1$ for all these models.

\begin{tabular}{lllllll}
\hline \hline$\Delta \theta$ & $\delta \alpha$ & $M$ & $M_{\mathrm{r}}$ & $M_{\theta}$ & $M_{\phi}$ & $E$ \\
\hline $45^{\circ}$ & 1 & 0.31 & 0.10 & 0.09 & 0.13 & 0.044 \\
$80^{\circ}$ & 1 & 0.38 & 0.11 & 0.12 & 0.15 & 0.052 \\
$80^{\circ}$ & 0.5 & 0.21 & 0.06 & 0.07 & 0.08 & 0.020 \\
\hline
\end{tabular}

magnetic field components to the total magnetic field strength. Obviously, $M=M_{\mathrm{r}}+M_{\theta}+M_{\phi}$. We also define $E$, the total magnetic energy.

The dominance of radial nonaxisymmetric structures at the surface, which is a consistent feature of the plots of surface magnetic field structures, is much less pronounced in the integral values, $M_{\mathrm{r}}, M_{\theta}, M_{\phi}$. We conclude again that it is $B_{\mathrm{r}}$ which demonstrates a tendency to give spotty nonaxisymmetric structure at the surface.

We note that $M$ does not become smaller, and even slightly increases, with $\left|C_{\omega}\right|$ for negative $C_{\omega}$. This rather counterintuitive result arises in part from the fact that dynamos with our strongly nonaxisymmetric alpha-distribution become less strongly excited as $\left|C_{\omega}\right|$ increases - see the $E$ values listed in Table 3. This feature is even more pronounced for $\theta$-dependent rotation laws (see below). The properties of magnetic spots demonstrate a moderate dependence on the other parameters 
governing the form and intensity for the $\alpha$-spot. Some relevant results are presented in Table 4.

\subsection{Rotation law $\Omega(r, \theta)$}

To test the robustness of our results, we also implemented a rotation law with a simple latitudinal dependence, $\Omega(r, \theta)=$ $C_{\omega}\left(r-r_{\omega}\right) \cos ^{2} \theta$, with $r_{\omega}=r_{0}$. This choice was to some extent motivated as follows. The strong tidal interaction possibly removes the region of superrotation which is observed near to the solar equator according to helioseismological data. Our simple rotation curve mimics very crudely such a rotation curve, but the main point is to demonstrate that our results do not depend on the assumption of purely radial rotational shear. The marginal value of $C_{\alpha}$ is again $22 \ldots 26$ when $\left|C_{\omega}\right| \lesssim 50$. Outline results are given in Table 5 .

One point in Table 5 deserves special attention (cf. also Table 3 with $C_{\omega}<0$ ). Surprisingly, when $C_{\omega}<0$, as $\left|C_{\omega}\right|$ increases, $M$ also increases, contrary to the usual interaction between differential rotation and a nonaxisymmetric magnetic field. However for $C_{\omega}=-1000$, the dynamo is not excited, and inspection of the $E$ values indicates that with increasing $\left|C_{\omega}\right|$ the dynamo becomes less supercritical, which favours nonaxisymmetric field generation in this model. The crucial point is that with our choice of alpha distribution, axisymmetic field generation is not favoured compared to generation of nonaxisymmetric fields - indeed the two cannot be separated.

The solutions outlined in the Table 5 are usually nonoscillatory with one exception, when $C_{\omega}=-25$. This solution demonstrates a remarkably high level of nonaxisymmetry; its oscillation period is $T \approx 0.54$.

\section{Resonant excitation}

For the computations described above, the surface of the dynamo region rotates with respect to the $\alpha$-spot, anchored to the base of the dynamo region at $r=r_{\omega}=r_{0}=0.64$. If the $\alpha$-spot is made to corotate with the fluid at depth $r_{\omega} \neq r_{0}$, then we found that the nonaxisymmetric field can be much enhanced. We put $\Omega \propto\left(r-r_{\omega}\right), \delta \alpha=1.0, C_{\omega}=50, C_{\alpha}=26, \Delta \theta=80^{\circ}$. The results are listed in Table 6 . The evolution with time of selected oscillatory solutions is shown in Fig. 4. Their spatial structure is presented in Fig. 5, and we show the variation of the global nonaxisymmetry $M$ with $r_{\omega}$ in Fig. 6 .

We conclude that for specific ranges of values of $r_{\omega}$ an excitation of oscillating nonaxisymmetric solutions is possible. The resonant value of $r_{\omega}$ is about 0.8 for the case presented in Table 6. However resonant excitation occurs for $r_{\omega}=r_{0}$ for the case presented in Table 5 when $C_{\alpha}=24, C_{\omega}=-25$. In a resonant solution, the level of nonaxisymmetry $M$ varies widely during the oscillation, and reaches very large values, in the range $0.92 \ldots 0.99$, at its maximum. The nonaxisymmetric solution is manifested on the stellar surface by a spot structure which again is most pronounced in the $B_{\mathrm{r}}$ distribution, and which looks similar to that in the nonresonant solution.

The results summarized in Table 6 were obtained with a rather strong $\alpha$-spot. We verified that our results are quite insensitive to the exact value of $\Delta \theta$ (e.g. $\Delta \theta=45^{\circ}$ gives very
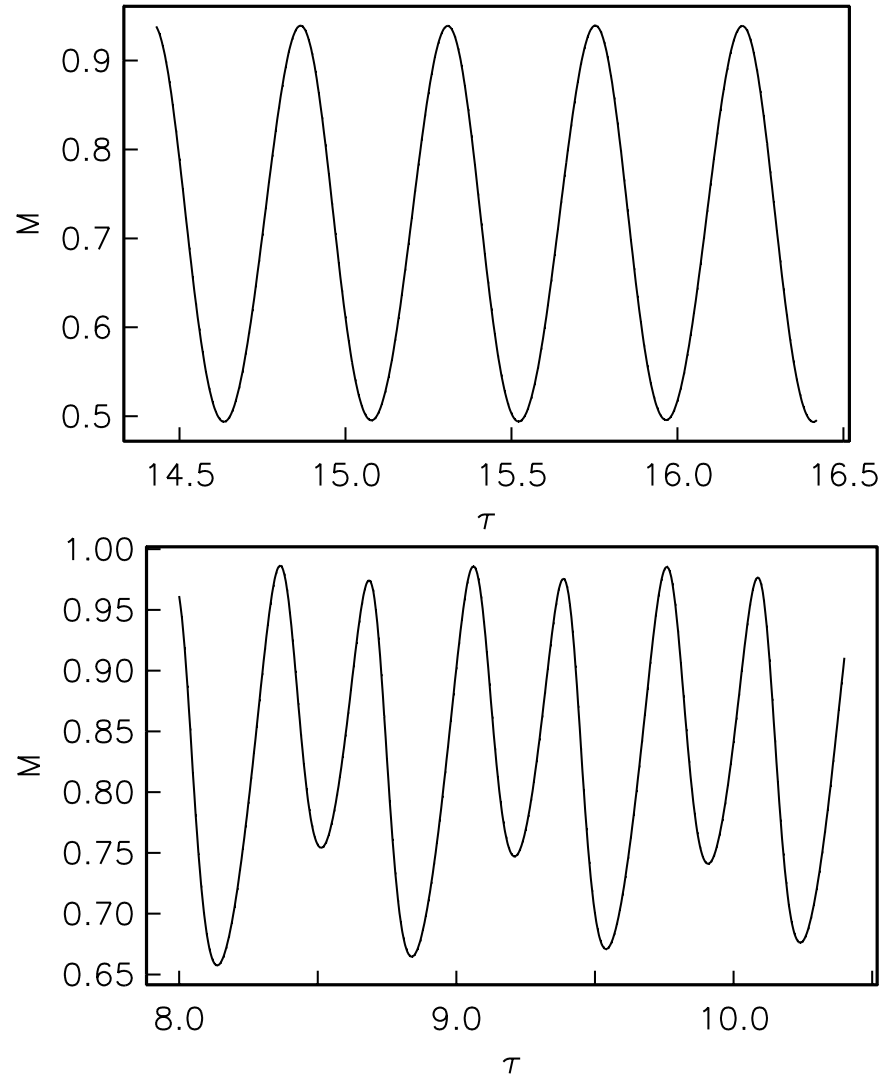

Fig. 4. Evolution with time of the global nonaxisymmetry parameter $M$, for the resonant solutions with $C_{\alpha}=26, \delta_{\alpha}=1$, and $C_{\omega}=50$, $\Delta \theta=80^{\circ}, r_{\omega}=0.78$ (top), and with $C_{\omega}=-50, \Delta \theta=45^{\circ}, r_{\omega}=0.76$ (bottom).

similar solutions) - this is readily understandable as the basic alpha profile becomes small near the poles. Also, reducing $\delta \alpha$ to 0.5 gives comparable resonant solutions. Thus our tabulated results do not apply only to rather extreme choices of parameters.

The oscillation periods of resonant solutions are about $T=$ $0.3 \ldots 0.5$ in dimensionless units. Taking for an estimate solarlike values for $\eta$, we obtain $T \approx 100 \mathrm{yr}$, which is much too long to be able to associate the observed variability of cool spots with the oscillatory regime. We need to assume that $\eta \approx 10 \eta \odot$ in order to get $T \approx 10 \mathrm{yr}$, which would be comparable to observed timescales.

\section{Discussion}

A very simple dynamo model which takes into account perturbations of the dynamo governing parameters in the rotating shell of one member of a close binary system from the influence of the other component, in form of an $\alpha$-spot, appears to be able to reproduce the basic features of cool spot distributions found on the stellar surface. These spots are thus attributed to the excitation of nonaxisymmetric magnetic structures by dynamo action. The cool spots can be associated with spots of radial mean magnetic field, which are displaced from the position of the $\alpha$-spot to higher latitudes. The $B_{\mathrm{r}}$-spots are displaced in longitude by about typically a dozen degrees from the $\alpha$-spot. This is comparable with the latitudinal displacement 
Table 5. Summary of results with $\Omega=C_{\omega}\left(r-r_{0}\right) \cos ^{2} \theta, \delta \alpha=1.0, \Delta \theta=45^{\circ}$. Oscillatory solutions are labelled by asterisks, and the range of variation of $M$ and $E$ are also indicated. $M_{\mathrm{r}}, M_{\theta}, M_{\phi}$ values for non-steady solutions are at an arbitrary time. $\phi$ is the central latitude of $B_{\mathrm{r}}$-spots.

\begin{tabular}{lllllllll}
\hline \hline$C_{\alpha}$ & $C_{\omega}$ & $M$ & $P$ & $E$ & $M_{r}$ & $M_{\theta}$ & $M_{\phi}$ & $\phi$ \\
\hline 50 & 250 & 0.03 & -1 & 1.06 & 0.007 & 0.007 & 0.017 & 25 \\
28 & 250 & 0.11 & -1 & 0.132 & 0.030 & 0.026 & 0.054 & 25 \\
28 & 100 & 0.19 & -1 & 0.107 & 0.053 & 0.043 & 0.089 & 18 \\
28 & 25 & 0.30 & -1 & 0.090 & 0.087 & 0.088 & 0.126 & 3 \\
24 & 25 & 0.49 & -1 & 0.027 & 0.14 & 0.14 & 0.21 & 3 \\
\hline $24^{*}$ & -25 & $0.60 \ldots 0.98$ & +1 & $0.015 \ldots 0.020$ & 0.25 & 0.32 & 0.40 & $-23,+23$ \\
28 & -100 & 0.15 & +1 & 0.053 & 0.15 & 0.32 & 0.40 & 0 \\
28 & -250 & 0.78 & +1 & 0.033 & 0.16 & 0.24 & 0.37 & -11 \\
28 & -1000 & 0.97 & +1 & decays & - & - & - & - \\
\hline
\end{tabular}
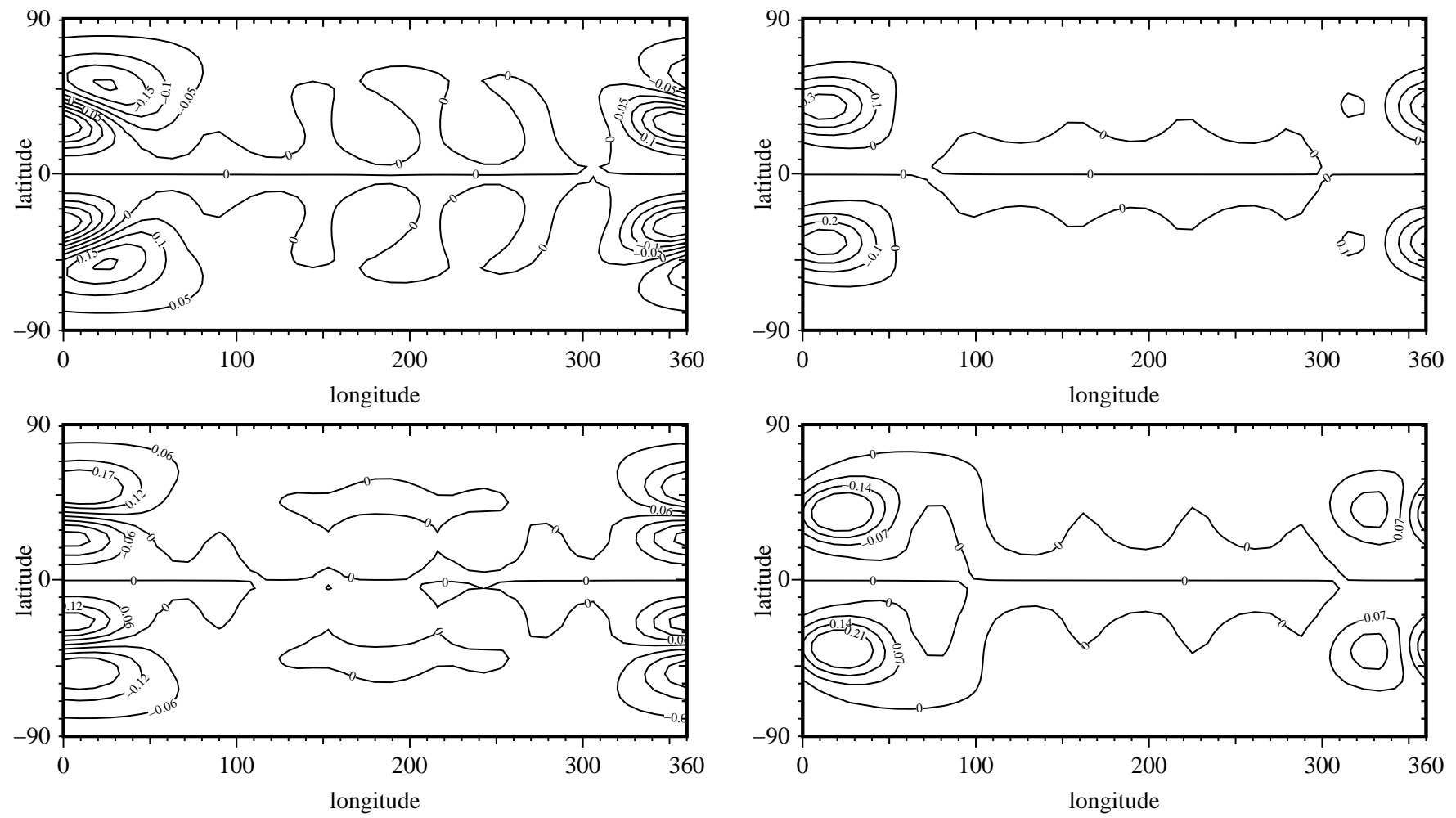

Fig. 5. Surface radial magnetic field structure for the resonant solution for $C_{\omega}=+50, r_{\omega}=0.79$. Top row, left $t=7.77$, right $t=7.98$; bottom row, left $t=8.23$, right $t=8.55$.

observed between the hot and cool spots. Sometimes several $B_{\mathrm{r}}$-spots are present in each hemisphere simultaneously; this is welcome given the variety of observational results. The spot sizes look comparable with those observed for cool spots. The latitudinal location of spots can be tuned by choosing an appropriate $\alpha$-profile; for example, if (instead of $\alpha_{0}=\sin ^{2} \theta \cos \theta$ ) we choose $\alpha_{0} \propto \cos \theta$, except very near to the poles, the spots are located in the subpolar regions.

The specific features of the hydrodynamics of close binaries are still quite obscure, and even the sign of the radial shear of the differential rotation, which is crucial for understanding the dynamo action, is unknown. We have focussed our attention on the magnetic field behaviour, leaving the hydrodynamical problem for future research. We have concentrated on finding models with dynamo excited spot configurations that can plausibly be compared with the observed starspots, and have considered simple models demonstrating the main physical effects involved. In this spirit, we have described the backreaction of the magnetic field on the stellar convection in terms of a conventional $\alpha$-quenching, appreciating that future developments of the theory should include a more sophisticated model of $\alpha$-limitation (see e.g. Kleeorin et al. 2000, 2002); there is also the further possibility of including the azimuthal component of the Lorentz force into the equation for the mean velocity (see e.g. Moss \& Brooke 2000). At this stage of the investigation, we consider the problem of possible identification of the resonant excitation found with a swing excitation, as suggested by Sokoloff \& Piskunov (2002), as a rather semantic question, and leave its interpretation for the future.

The prediction of the dominance of radial magnetic fields in nonaxisymmetric structures appears to be in agreement with the limited observational evidence. Donati (1999) seems 
Table 6. Summary of results with $\Omega=C_{\omega}\left(r-r_{\omega}\right), \delta \alpha=1.0, C_{\omega}= \pm 50, C_{\alpha}=26, \Delta \theta=80^{\circ}$, as $r_{\omega}$ varies. Oscillatory solutions are indicated by asterisks and $T$ is then the oscillation period of the total energy $E . \phi$ is the approximate central longitude of the dominant $B_{\mathrm{r}}$-spots. Solutions with double asterisks are doubly periodic in $M$, and then $T$ is the mean period. The case $C_{\omega}=+50, r_{\omega}=0.88$ initially has a multiply periodic oscillation, with a very long transient phase, before settling to a singly periodic state.

\begin{tabular}{|c|c|c|c|c|c|c|c|c|}
\hline$r_{\omega}$ & $M$ & $P$ & $M_{\mathrm{r}}$ & $M_{\theta}$ & $M_{\phi}$ & $E$ & $T$ & $\phi$ \\
\hline \multicolumn{9}{|c|}{$C_{\omega}=+50$} \\
\hline 0.0 & 0.14 & -1.0 & 0.03 & 0.04 & 0.06 & 0.023 & - & 49 \\
\hline 0.64 & 0.38 & -1.0 & 0.11 & 0.12 & 0.15 & 0.052 & - & 18 \\
\hline 0.70 & 0.43 & -1.0 & 0.12 & 0.15 & 0.15 & 0.054 & - & 8 \\
\hline 0.76 & 0.55 & -1.0 & 0.16 & 0.23 & 0.17 & 0.052 & - & 0 \\
\hline $0.78^{*}$ & $0.50 \ldots 0.94$ & -1.0 & 0.16 & 0.12 & 0.21 & $0.044 \ldots 0.049$ & 0.45 & 15 \\
\hline $0.785^{* *}$ & $0.50 \ldots 0.94$ & -1.0 & 0.16 & 0.21 & 0.21 & $0.045 \ldots 0.049$ & 0.45 & 15 \\
\hline $0.79^{*}$ & $0.42 \ldots 0.96$ & -1.0 & 0.21 & 0.16 & 0.32 & $0.040 \ldots 0.054$ & 0.49 & 25 \\
\hline $0.82^{*}$ & $0.44 \ldots 0.93$ & -1.0 & 0.27 & 0.30 & 0.34 & $0.047 \ldots 0.055$ & 0.35 & 12 \\
\hline $0.88^{*}$ & $0.66 \ldots 0.92$ & -1.0 & 0.28 & 0.32 & 0.33 & $0.047 \ldots 0.049$ & 0.33 & 6 \\
\hline 0.91 & 0.48 & -1.0 & 0.16 & 0.12 & 0.22 & 0.049 & - & -11 \\
\hline 0.94 & 0.42 & -1.0 & 0.14 & 0.09 & 0.19 & 0.049 & - & -12 \\
\hline 1.00 & 0.37 & -1.0 & 0.12 & 0.09 & 0.16 & 0.055 & - & -16 \\
\hline \multicolumn{9}{|c|}{$C_{\omega}=-50$} \\
\hline 0.64 & 0.48 & +1 & 0.12 & 0.19 & 0.17 & 0.038 & - & -23 \\
\hline 0.70 & 0.57 & +1 & 0.14 & 0.23 & 0.20 & 0.037 & - & -23 \\
\hline 0.75 & 0.76 & +1 & 0.18 & 0.30 & 0.28 & 0.030 & - & -30 \\
\hline $0.76^{* *}$ & $0.58 \ldots 0.99$ & +1 & 0.27 & 0.27 & 0.36 & $0.024 \ldots 0.037$ & 0.35 & -17 \\
\hline $0.77^{*}$ & $0.66 \ldots 0.98$ & +1 & 0.25 & 0.31 & 0.40 & $0.030 \ldots 0.036$ & 0.38 & -24 \\
\hline $0.80^{*}$ & $0.56 \ldots 0.97$ & +1 & 0.17 & 0.19 & 0.20 & $0.025 \ldots 0.039$ & 0.44 & -10 \\
\hline $0.82^{*}$ & $0.59 \ldots 0.96$ & +1 & 0.26 & 0.29 & 0.39 & $0.026 \ldots 0.036$ & 0.43 & -10 \\
\hline 0.85 & 0.74 & +1 & 0.23 & 0.19 & 0.32 & 0.034 & - & 10 \\
\hline 0.88 & 0.60 & +1 & 0.19 & 0.15 & 0.26 & 0.041 & - & 8 \\
\hline 0.94 & 0.50 & +1 & 0.16 & 0.13 & 0.21 & 0.044 & - & 12 \\
\hline 1.0 & 0.45 & +1 & 0.14 & 0.12 & 0.19 & 0.036 & - & 18 \\
\hline
\end{tabular}

to detect circular polarization for a number of short period binaries (RS CVn systems, e.g. HR 1099) but only for a very short fraction of the period, typically at one phase. This would be the case if there is a dominant radial magnetic field at the subpolar region.

Our solutions demonstrate a deep coupling between the sign of differential rotation ( $\operatorname{sign}$ of $C_{\omega}$ ) and the solution parity; solutions with $C_{\omega}>0$ have structures with odd parity radial fields with respect to the rotational equator, whereas solutions with $C_{\omega}<0$ have basically an even parity of the radial field. This behaviour means that nonuniform part of the rotation influences substantially the solutions obtained.

One further point of potential interest is that solutions in or near the resonant regimes typically have long transients and, for example, solutions in singly periodic regimes may appear doubly periodic for tens of oscillation periods. This suggests that any physical perturbation (e.g. to the large scale convection) may initiate a significant episode of nonsteady behaviour.

A natural way to improve the observational basis of our model would be a comparative observational progamme for binary systems suitable for surface imaging with various separations between companions. Such a programme could isolate in a more detailed way the link between the stellar separation and the level of nonaxisymmetry for these systems. We address the reader again to the analysis undertaken by Hall (1991), whilst observing that even if a reduction in differential rotation is the only systematic effect with decreasing separation of components, then in general this could be expected to promote generation of nonaxisymmetric fields (although there are certainly circumstances, as demonstrated above, where this is not necessarily the case).

The large amplitude oscillatory solutions, which can be considered as a resonant excitation of an oscillating dynamo mode, appear to be quite different from the conventional oscillating mode of the Parker migratory dynamo, and give spots with oscillating intensity located at fixed latitudes, rather than migrating latitudinally during the cycle. We obtain a cycle period which is rather long, for standard values of the parameters. On one hand, this discrepancy is of the same order as that for the solar cycle period as found in early models for the solar dynamo (of course the calculated periods for solar dynamo were too short, and here they are too long). On the other hand, this discrepancy can be removed by invoking a larger value of the turbulent diffusivity in the convective shells of close binaries, compared with the solar case. 

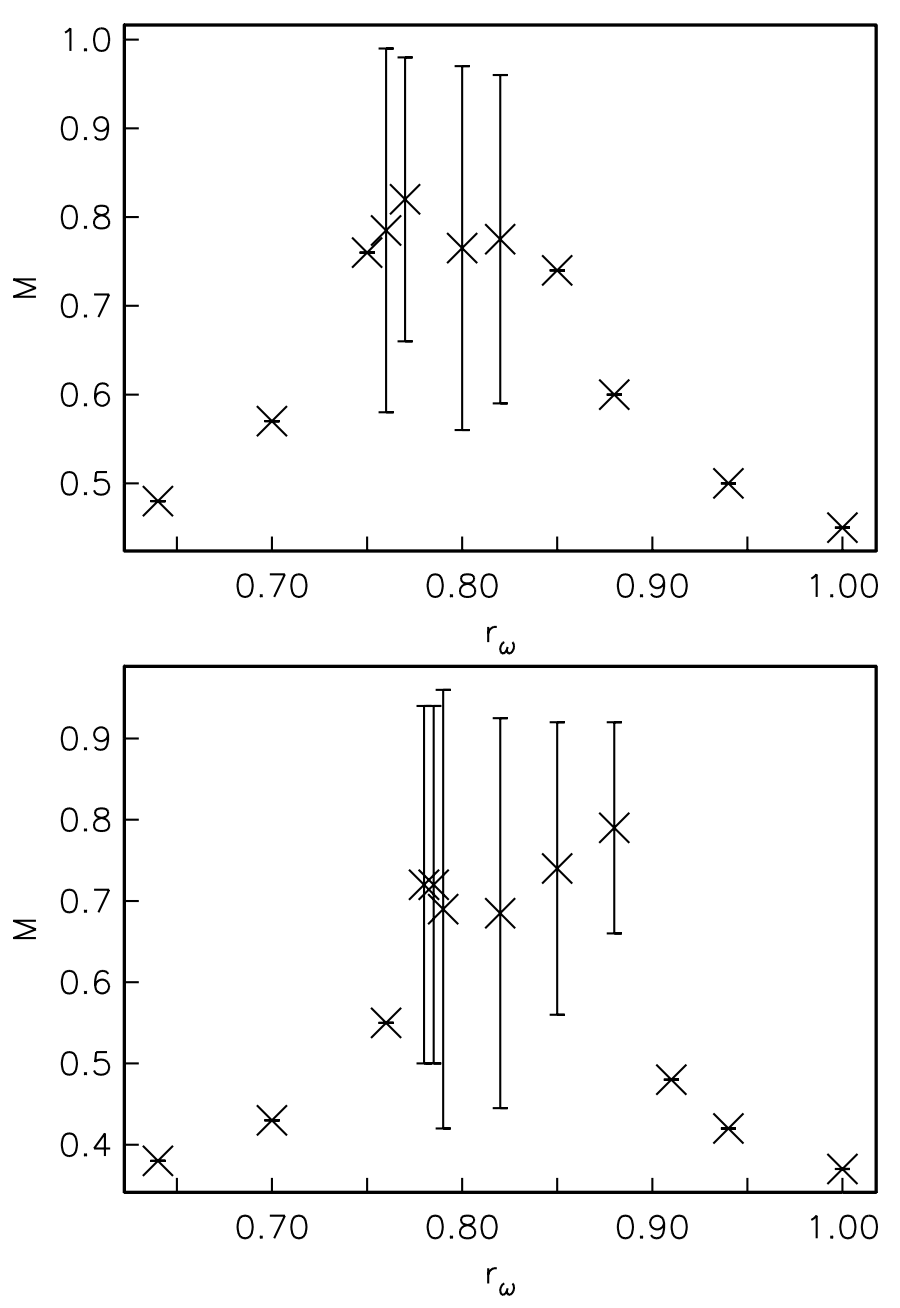

Fig. 6. Variation of the global nonaxisymmetry measure $M$ with $r_{\omega}$ for the models summarized in Table 6. For oscillatory solutions, the range about the mean is indicated. Top panel is for $C_{\omega}=-50$, lower for $C_{\omega}=+50$.

The similarity of the simulated magnetic spots to the cool spots observed on ER Vulpeculae can be demonstrated by comparing the spot structure of the $B_{\mathrm{r}}$ field for a resonant case at its maximal phase $\left(r_{\omega}=0.79\right.$ listed in Table 6$)$ and the spot structure of the primary star of EU Vulpeculae in the 1996 observing season at phase $\phi=0.000$ (Fig. 1 in Sokoloff \& Piskunov 2002). We note however that the observed hot spot is located above the equator, making the whole picture asymmetric with respect to the equator, and thus suggesting the need for additional elaboration of the theory.

Our $\alpha$-enhancement mechanism is heuristic, and in principle we cannot rule out the possibility that $\alpha$ might instead be suppressed near the sub-companion longitude. Preliminary numerical experiments suggest that $B_{\mathrm{r}}$-spots are then found near longitude $\phi=180^{\circ}$. Our limited experimentation did not find resonant solutions, but we cannot dismiss the possibility.

We note that the observational basis for the conclusion that oscillatory dynamo solutions are required to explain the temporal behaviour of observed spots is very limited. In principle, the variability of cool spots could be attributed to the processes of spot formation by a steady nonaxisymmetric magnetic field distribution. A natural long-term approach to a better understanding of this topic would be a long timescale observational programme for a sample of close binaries, comparable to the well-known observational programme for the Wilson sample (see e.g. Baliunas et al. 1995). Comparative studies of binaries with various separations and long-term monitoring of a limited sample of similar binaries can be considered to be comparable approaches.

We also note that standard models of stellar convection do not take into account external forcing from the companion star and so the usual estimates of convective parameters may need reconsideration.

Acknowledgements. DS and DM are grateful to Uppsala University for its hospitality. DS also thanks the Royal Swedish Academy of Sciences for financial support during his visit to Sweden and to the Royal Society, London during his visit to the UK. Financial support from NATO (grant PST.CLG 974737), and for DS from RFBR (grant 01-02-17693) is acknowledged. We thank the referee for a helpful report.

\section{References}

Baliunas, S., Donahue, R. A., Soon, W. H., et al. 1995, ApJ, 438, 269 Berdyugina, A. V., Ilyin, I., \& Tuominen, I. 1999, A\&A, 350, 626

Donati, J.-F. 1999, MNRAS, 302, 457

Gunn, A. G., \& Doyle, J. G. 1997, A\&A, 318, 60

Hall, D. S. 1991, in The Sun and Cool Stars: Activity, Magnetism, Dynamos, ed. I. Tuominen, D. Moss, \& G. Rüdiger (Berlin: Springer), 352

Henry, G. W., Eaton, J. A., Hamer, J., \& Hall, D. S. 1995, ApJS, 97, 513

Jetsu, L., Pohjolainen, S., Pelt, J., \& Tuominen, I. 1997, A\&A, 318, 293

Kleeorin, N., Moss, D., Rogachevskii, I., \& Sokoloff, D. 2000, A\&A, 361, L5

Kleeorin, N., Moss, D., Rogachevskii, I., \& Sokololoff, D. 2002, A\&A, 387, 453

Krause, F., \& Rädler, K.-H. 1980, Mean-field Magnetohydrodynamics and Dynamo Theory (Oxford: Pergamon)

Moss, D. L., Mestel, L., \& Tayler, R. J. 1990, MNRAS, 245, 440

Moss, D., Brandenburg, A., \& Tuominen, I. 1991a, A\&A, 247, 576

Moss, D., \& Brooke, J. 2000, MNRAS, 315, 521

Moss, D., \& Tuominen, I. 1997, A\&A, 321, 151

Moss, D., Tuominen, I., \& Brandenburg, A. 1991b, A\&A, 245, 129

Olah, K., Koevari, Zs., Bartus, J., et al. 1997, A\&A, 321, 811

Piskunov, N. 1996, in Stellar Surface Structure, ed. K. G. Strassmeier, \& J. S. Linsky (Dordrecht: Kluwer), Proc. IAU Symp., 176, 45

Piskunov, N. E., Tuominen, I., \& Vilhu, O. 1990, A\&A, 294, 155

Piskunov, N., Vincent, A., Duemmler, R., Ilyin, I., \& Tuominen, I. 2001, in 11th Cambridge Workshop on Cool Stars, Stellar Systems and the Sun, Tenerife 1999, ed. R. J. García Lopez, R. Rebolo, \& M. R. Zapatero Orosoi, ASP Conf. Ser., 223, CD-1285

Sokoloff, D., \& Piskunov, N. 2002, MNRAS, 334, 925

Vogt, S. S., Harzes, A. P., Misch, A. A., \& Kürster, M. 1999, ApJ, 121, 547 